\title{
VIABILITY FOR A NONLINEAR MULTI-VALUED SYSTEM ON LOCALLY CLOSED GRAPH
}

\author{
BY
}

\section{DANIELA ROŞU}

\begin{abstract}
The purpose of this paper is to prove some necessary and sufficient conditions in order that the graph $\mathcal{K}$ of the multi-function $K: I \rightsquigarrow \overline{D(A)} \times \overline{D(B)}$ be $C^{0}$-viable with respect to the nonlinear system of the form

$$
\begin{cases}u^{\prime}(t) \in A u(t)+F(t, u(t), v(t)), & t \geq \tau \\ v^{\prime}(t) \in B v(t)+G(t, u(t), v(t)), & t \geq \tau \\ u(\tau)=\xi, v(\tau)=\eta, & \end{cases}
$$

where $I \subseteq \mathbb{R}$ is an open from the right interval, $X$ and $Y$ are real Banach spaces, $A$ : $D(A) \subseteq X \rightsquigarrow X$ and $B: D(B) \subseteq Y \rightsquigarrow Y$ are $m$-dissipative operators generating nonlinear semigroups of contractions, $F: \mathcal{K} \rightarrow X$ is a given function and $G: \mathcal{K} \rightsquigarrow Y$ is a nonempty valued multi-function. We provide a necessary and sufficient condition in order that the system has at least one $C^{0}$-solution $(u, v)$ satisfying time-dependent constrains $(u(t), v(t)) \in K(t)$ for each $t$. We include a comparison result referring to a nonlinear system with multi-valued perturbations of subdifferentials in a Hilbert space.

Mathematics Subject Classification 2000: 35K57, 34G25, 47J35, 47H06, 47H20.

Key words: $m$-dissipative operator, reaction-diffusion system, viable set, tangency condition.
\end{abstract}

\section{Introduction}

Let $\left(X,\|\cdot\|_{X}\right)$ and $\left(Y,\|\cdot\|_{Y}\right)$ be real Banach spaces and let $A: D(A) \subseteq$ $X \rightsquigarrow X$ and $B: D(B) \subseteq Y \rightsquigarrow Y$ be $m$-dissipative operators generating the nonlinear semigroups of contractions, $\left\{S_{A}(t): \overline{D(A)} \rightarrow \overline{D(A)} ; t \geq 0\right\}$ and $\left\{S_{B}(t): \overline{D(B)} \rightarrow \overline{D(B)} ; t \geq 0\right\}$ respectively. Let $I \subseteq \mathbb{R}$ be a nonempty and open from the right interval, let $K: I \rightsquigarrow \overline{D(A)} \times \overline{D(B)}$ be a given nonempty valued multi-function and $\mathcal{K}:=\operatorname{graph}(K)$. 
We consider the system

$$
\left\{\begin{array}{l}
u^{\prime}(t) \in A u(t)+F(t, u(t), v(t)), \quad t \geq \tau \\
v^{\prime}(t) \in B v(t)+G(t, u(t), v(t)), \quad t \geq \tau \\
u(\tau)=\xi, \quad v(\tau)=\eta
\end{array}\right.
$$

where $(\tau, \xi, \eta) \in \mathcal{K}, F: \mathcal{K} \rightarrow X$ is a given function and $G: \mathcal{K} \rightsquigarrow Y$ is a multi-function with nonempty values.

We are interested in finding necessary and sufficient conditions in order that $\mathcal{K}$ be $C^{0}$-viable with respect to $(A+F, B+G)$.

Definition 1.1. The continuous function $(u, v):[\tau, T] \rightarrow \overline{D(A)} \times \overline{D(B)}$ is a $C^{0}$-solution of $(1.1)$ on $[\tau, T]$ if $(t, u(t), v(t)) \in \mathcal{K}$ for each $t \in[\tau, T]$, the function $f(t)=F(t, u(t), v(t))$ for each $t \in[\tau, T]$, belongs to $L^{1}(\tau, T ; X)$ and there exists $g \in L^{1}(\tau, T ; Y)$ such that $g(t) \in G(t, u(t), v(t))$ a.e. for $t \in[\tau, T]$ and $(u, v)$ is a $C^{0}$-solution on $[\tau, T]$ of the problem

$$
\left\{\begin{array}{l}
u^{\prime}(t) \in A u(t)+f(t), \\
v^{\prime}(t) \in B v(t)+g(t), \\
u(\tau)=\xi, \quad v(\tau)=\eta .
\end{array}\right.
$$

Definition 1.2. The graph, $\mathcal{K}$, of $K: I \rightsquigarrow \overline{D(A)} \times \overline{D(B)}$, is $C^{0}$-viable with respect to $(A+F, B+G)$ if for each $(\tau, \xi, \eta) \in \mathcal{K}$ there exists $T>\tau$, $[\tau, T] \subseteq I$ such that the problem (1.1) has at least one $C^{0}$-solution on $[\tau, T]$.

A growing literature is devoted to the abstract theory of viability referring to the differential inclusion $u^{\prime}(t) \in A u(t)+F(t, u(t))$. We mention the starting paper of PAVEL [15] for the semilinear single-valued case and VRABIE [19] for nonlinear case. Referring to the semilinear multi-valued case when $A$ is linear unbounded, $K \subseteq I \times X$ is locally closed set and $F: K \rightsquigarrow X$ is multi-valued, we notice the works of PAVEL-VRABIE [16], [17], CÂrJă-Vrabie [11], [10], CÂrJă-Necula-Vrabie [8]. As concerns the fully nonlinear inclusion see Bothe [2], [3], CÂRJ $\breve{A}-$ NeCUla-Vrabie [7],[9]. For the case in which $F$ is defined on the graph $\mathcal{K}$, of the multi-function $K: I \rightsquigarrow \overline{D(A)}$ see Necula-Popescu-Vrabie [13], [14]. They introduced the concept of $A$-quasi-tangent set to the graph of $K$ at a given point $(\tau, \xi) \in \mathcal{K}$ in order to give a necessary and sufficient condition for 
$C^{0}$-viability referring to the nonlinear inclusion with multi-valued and $t$ discontinuous perturbations defined on graphs. We recall this concept and the necessary condition for $C^{0}$-viability in section 2 .

The $C^{0}$-viability problem of a locally closed set with respect to the multi-valued reaction-diffusion system of the form (1.1) has been studied by BURLICA $\breve{A}$ [4] in the semilinear case and by RoşU [18] in the nonlinear case. The semilinear system with multi-valued perturbations defined on graphs has been considered by BURLIC $\breve{A}$ [5].

Our paper is divided into 5 sections. In Section 2 we recall some results referring to evolutions governed by $m$-dissipative operators, the concept of tangent sets and some concepts about multi-functions. The third section contains the main results for $C^{0}$-viability concerning the system (1.1), while in Section 4 we prove the main sufficient condition. In Section 5, as an application of our viability results, we give a comparison result referring to a nonlinear system with multi-valued perturbations of subdifferentials in a Hilbert space.

\section{Preliminaries}

In that follows, $(X,\|\cdot\|)$ denotes a real Banach space. For $\xi \in X$ and $\rho>0, D(\xi, \rho)$ denotes the closed ball in $X$ of radius $\rho$ centered in $\xi$ and $S(\xi, \rho)$ denotes the corresponding open ball. For $x \in X, C \subseteq X$ and $E \subseteq X$, we denote

$\|C\|=\sup _{x \in C}\|x\|, \operatorname{dist}(x, C)=\inf _{y \in C}\|x-y\|$ and $\operatorname{dist}(E, C)=\inf _{(x, y) \in E \times C}\|x-y\|$.

Let $A: D(A) \subseteq X \rightsquigarrow X$ be an $m$-dissipative operator, $\xi \in \overline{D(A)}$ and $f \in L^{1}(\tau, T ; X)$ and let us consider the Cauchy problem:

$$
\left\{\begin{array}{l}
u^{\prime}(t) \in A u(t)+f(t), \quad t \geq \tau \\
u(\tau)=\xi
\end{array}\right.
$$

Definition 2.1. A $C^{0}$-solution of the problem (2.1) is a continuous function $u \in C([\tau, T] ; X)$ satisfying $u(\tau)=\xi$ and, for each $\tau<c<T$ and $\varepsilon>0$ there exist

(i) $\tau=t_{0}<t_{1}<\cdots<c \leq t_{n}<T, t_{k}-t_{k-1} \leq \varepsilon$ for $k=1,2, \ldots, n$;

(ii) $f_{1}, \ldots, f_{n} \in X$ with $\sum_{k=1}^{n} \int_{t_{k-1}}^{t_{k}}\left\|f(t)-f_{k}\right\| d t \leq \varepsilon$; 
(iii) $v_{0}, \ldots, v_{n} \in X$ satisfying:

$$
\begin{gathered}
\frac{v_{k}-v_{k-1}}{t_{k}-t_{k-1}} \in A v_{k}+f_{k} \text { for } k=1,2, \ldots, n \\
\left\|u(t)-v_{k}\right\| \leq \varepsilon \text { for } t \in\left[t_{k-1}, t_{k}\right), k=1,2, \ldots, n .
\end{gathered}
$$

Theorem 2.1. Let $A: D(A) \subseteq X \rightsquigarrow X$ be m-dissipative. Then, for each $\xi \in \overline{D(A)}$ and $f \in L^{1}(\tau, T ; X)$, there exists a unique $C^{0}$-solution $u:[\tau, T] \rightarrow \overline{D(A)}$ of the problem $(2.1)$.

See Lakshmikantham-Leela [12, Theorem 3.6.1, p. 116].

We denote by $u(\cdot, \tau, \xi, f):[\tau, T] \rightarrow \overline{D(A)}$ the unique $C^{0}$-solution of (2.1) satisfying $u(\tau, \tau, \xi, f)=\xi$. We recall that, if $\widetilde{u}=u(\cdot, \tau, \xi, f)$ and $\widetilde{v}=u(\cdot, \tau, \eta, g)$ we have

$$
\|\widetilde{u}(t)-\widetilde{v}(t)\| \leq\|\xi-\eta\|+\int_{\tau}^{t}\|f(s)-g(s)\| d s,
$$

for each $t \in[\tau, T]$. Moreover, for each $\tau \leq a \leq \nu \leq t \leq \nu+\delta$, we have the following evolution property $u(t, a, \xi, f)=u\left(t, \nu, u(\nu, a, \xi, f),\left.f\right|_{[\nu, \nu+\delta]}\right)$. See VRABIE [20, Corollary 1.7.1, p. 26].

If $A: D(A) \subseteq X \rightsquigarrow X$ is $m$-dissipative, $\{S(t): \overline{D(A)} \rightarrow \overline{D(A)} ; t \geq 0\}$ denotes the nonlinear semigroup of nonexpansive mappings generated by $A$ on $\overline{D(A)}$, i.e. $S(t) \xi=u(t, 0, \xi, 0)$ for each $t \geq 0$ and $\xi \in \overline{D(A)}$.

For more details referring to evolutions governed by $m$-dissipative operators, see Barbu [1], Lakshmikantham-Leela [12] or Vrabie [20].

Now, let us consider the Cauchy problem

$$
\left\{\begin{array}{l}
u^{\prime}(t) \in A u(t)+F(t, u(t)), \\
u(\tau)=\xi,
\end{array}\right.
$$

where $A: D(A) \subseteq X \rightsquigarrow X$ is $m$-dissipative, $I \subseteq \mathbb{R}$ is a nonempty and open from the right interval, $K: I \rightsquigarrow \overline{D(A)}$ and $F: \mathcal{K} \rightsquigarrow X$ are two multifunctions with nonempty values and $\mathcal{K} \subseteq I \times \overline{D(A)}$ is the graph of $K$, i.e. $\mathcal{K}=\{(t, u) ; u \in K(t)\}$.

Definition 2.2. The continuous function $u:[\tau, T] \rightarrow X$ is a $C^{0}$ solution of the problem $(2.3)$ if $(t, u(t)) \in \mathcal{K}$ for each $t \in[\tau, T]$, there exists $f \in L^{1}(\tau, T ; X)$, with $f(s) \in F(s, u(s))$ a.e. for $s \in[\tau, T]$ and $u$ is a $C^{0}$-solution on $[\tau, T]$ of the problem (2.1) in the sense of Definition 2.1. 
Definition 2.3. The graph $\mathcal{K}$ is $C^{0}$-viable with respect to $A+F$ if for each $(\tau, \xi) \in \mathcal{K}$ there exists $T>\tau,[\tau, T] \subseteq I$ such that (2.3) has at least one $C^{0}$-solution $u:[\tau, T] \rightarrow X$.

We recall the concepts of $A$-tangent set and $A$-quasi-tangent set introduced by CÂRJ $\breve{A}$-NECUlA-VRABIE [6] in the constant case, i.e. $K(t)=K$ for each $t \in I$, and by Necula-Popescu-VRABie [14] in the general case. Let $(\tau, \xi) \in \mathcal{K}$ and let $E$ be a nonempty and bounded subset in $X$.

Definition 2.4. We say that the set $E \subseteq X$ is

(i) A-tangent to $\mathcal{K}$ at the point $(\tau, \xi)$ if

$$
\liminf _{h \downarrow 0} \frac{1}{h} \operatorname{dist}(u(\tau+h, \tau, \xi, E) ; K(\tau+h))=0,
$$

where

$$
u(\tau+h, \tau, \xi, E)=\{u(\tau+h, \tau, \xi, \eta) ; \eta \in E\} ;
$$

(ii) A-quasi-tangent to $\mathcal{K}$ at the point $(\tau, \xi)$ if

$$
\liminf _{h \downarrow 0} \frac{1}{h} \operatorname{dist}\left(u\left(\tau+h, \tau, \xi, \mathcal{F}_{E}\right) ; K(\tau+h)\right)=0,
$$

where $u\left(\tau+h, \tau, \xi, \mathcal{F}_{E}\right)=\left\{u(\tau+h, \tau, \xi, f) ; f \in L_{\text {loc }}^{1}(\mathbb{R} ; X), f(s) \in E\right.$ a.e. for $s \in \mathbb{R}\}$.

We denote by $\mathcal{T}_{\mathcal{K}}^{A}(\tau, \xi)$ the class of all $A$-tangent sets to $\mathcal{K}$ at $(\tau, \xi) \in \mathcal{K}$ and by $\mathcal{Q T S}_{\mathcal{K}}^{A}(\tau, \xi)$ the class of all $A$-quasi-tangent sets to $\mathcal{K}$ at $(\tau, \xi) \in \mathcal{K}$. Obviously, we have $\mathcal{T S}_{\mathcal{K}}^{A}(\tau, \xi) \subseteq 2 \mathcal{T} \mathcal{S}_{\mathcal{K}}^{A}(\tau, \xi)$.

Next, we recall some basic concepts and results from NECULA-POPESCUVRABIE [14], we need in the sequel. Throughout, $\lambda$ is the Lebesgue measure on $\mathbb{R}$.

Definition 2.5. The multi-function $F: \mathcal{K} \rightsquigarrow X$ is called (stronglyweakly) almost u.s.c. if, for each $\varepsilon>0$, there exists an open set $\mathcal{O}_{\varepsilon} \subseteq I$ such that $\lambda\left(\mathcal{O}_{\varepsilon}\right) \leq \varepsilon$ and $\left.F\right|_{\left[\left(I \backslash \mathcal{O}_{\varepsilon}\right) \times X\right] \cap \mathcal{K}}$ is (strongly-weakly) u.s.c.

Definition 2.6. The multi-function $F: \mathcal{K} \rightsquigarrow X$ is called essentially locally bounded if, for each $(\tau, \xi) \in \mathcal{K}$, there exist a negligible set $N_{1} \subset I, \rho>$ 0 and $l_{1} \in L_{\text {loc }}^{\infty}(I ; \mathbb{R})$ such that for all $(t, u) \in\left(\left(I \backslash N_{1}\right) \times D(\xi, \rho)\right) \cap \mathcal{K}$, we have $\|F(t, u)\| \leq l_{1}(t)$. If the same condition is satisfied with $l_{1} \in L_{\text {loc }}^{1}(I ; \mathbb{R})$, we say that $F$ is locally integrally bounded. 
Remark 2.1. If $D(A)$ is separable, we can choose $N_{1}$ in Definition 2.6 independent of $(\tau, \xi) \in \mathcal{K}$.

Definition 2.7. The graph $\mathcal{K}$ is $A-C^{0}$-viable by itself if for each $(\tau, \xi) \in$ $\mathcal{K}$ there exist $T>\tau, \rho>0$ and $l_{2} \in L_{\text {loc }}^{1}(I ; \mathbb{R})$, so that for each $(\widetilde{\tau}, \widetilde{\xi}) \in$ $([\tau, T) \times S(\xi, \rho)) \cap \mathcal{K}$, there exist $\widetilde{T} \in(\widetilde{\tau}, T]$ and a pair of functions, $(g, v) \in L^{1}([\widetilde{\tau}, \widetilde{T}] ; X) \times C([\widetilde{\tau}, \widetilde{T}] ; X)$, satisfying:

(i) $v(t)=u(t, \widetilde{\tau}, \widetilde{\xi}, g)$ for each $t \in[\widetilde{\tau}, \widetilde{T}]$;

(ii) $(t, v(t)) \in([\widetilde{\tau}, \widetilde{T}] \times S(\xi, \rho)) \cap \mathcal{K}$, for each $t \in[\widetilde{\tau}, \widetilde{T}]$;

(iii) $\|g(s)\| \leq l_{2}(s)$ a.e. for $s \in[\widetilde{\tau}, \widetilde{T}]$.

A pair $(g, v)$ satisfying (i)-(iii) is called simple solution issuing from $(\widetilde{\tau}, \widetilde{\xi}) \in([\tau, T) \times S(\xi, \rho)) \cap \mathcal{K}$.

Remark 2.2. (i) If $K: I \rightsquigarrow \overline{D(A)}$ is constant and $S(t) K \subseteq K$ for each $t \geq 0$, then $\mathcal{K}$ is $A-C^{0}$-viable by itself.

(ii) If $\mathcal{K}$ is $C^{0}$-viable with respect to $A+F$, where $F: \mathcal{K} \rightsquigarrow X$ is essentially locally bounded multi-function, then $\mathcal{K}$ is $A$ - $C^{0}$-viable by itself.

Definition 2.8. (i) The graph $\mathcal{K}$ is locally closed from the left if for each $(\tau, \xi) \in \mathcal{K}$ there exist $T>\tau$ and $\rho>0$ such that, for each $\left(\tau_{n}, \xi_{n}\right) \in$ $([\tau, T] \times D(\xi, \rho)) \cap \mathcal{K}$, with $\left(\tau_{n}\right)_{n}$ nondecreasing, $\lim _{n} \tau_{n}=\widetilde{\tau}$ and $\lim _{n} \xi_{n}=$ $\widetilde{\xi}$, we have $(\widetilde{\tau}, \widetilde{\xi}) \in \mathcal{K}$;

(ii) The graph $\mathcal{K}$ is closed from the left if for each $\left(\tau_{n}, \xi_{n}\right) \in \mathcal{K}$, with $\left(\tau_{n}\right)_{n}$ nondecreasing, $\lim _{n} \tau_{n}=\widetilde{\tau}$ and $\lim _{n} \xi_{n}=\widetilde{\xi}$, we have $(\widetilde{\tau}, \widetilde{\xi}) \in \mathcal{K}$.

Definition 2.9. An $m$-dissipative operator $A: D(A) \subseteq X \rightsquigarrow X$ is of compact type if for each sequence $\left(\left(f_{n}, u_{n}\right)\right)_{n}$ in $L^{1}(\tau, T ; X) \times C([\tau, T] ; X)$ with $u_{n}$ a $C^{0}$-solution of the problem $u_{n}^{\prime}(t) \in A u_{n}(t)+f_{n}(t)$ on $[\tau, T]$ for $n=1,2, \ldots, \lim _{n} f_{n}=f$ weakly in $L^{1}(\tau, T ; X)$ and $\lim _{n} u_{n}=u$ strongly in $C([\tau, T] ; X)$, it follows that $u$ is a $C^{0}$-solution of the problem $u^{\prime}(t) \in$ $A u(t)+f(t)$ on $[\tau, T]$.

We conclude this section with a necessary condition for $C^{0}$-viability referring to the inclusion (2.3).

Theorem 2.2. Let $X$ be a Banach space, let $A: D(A) \subseteq X \rightsquigarrow X$ be an $m$-dissipative operator which generates a nonlinear semigroup of contractions on $\overline{D(A)}$ and let $F: \mathcal{K} \rightsquigarrow X$ be a multi-function with nonempty 
and closed values, almost u.s.c. and locally integrally bounded. If $D(A)$ is separable and $\mathcal{K}$ is $C^{0}$-viable with respect to $A+F$, then $\mathcal{K}$ is $A$ - $C^{0}$-viable by itself and there exists a set $N \subseteq I$, with $\lambda(N)=0$, such that, for each $(\tau, \xi) \in[(I \backslash N) \times X] \cap \mathcal{K}$, we have $F(\tau, \xi) \in \mathcal{Q T}_{\mathcal{T}}^{A}(\tau, \xi)$.

See Necula-Popescu-Vrabie [14, Theorem 4.1].

\section{The main results}

The aim of this section is to present the necessary and sufficient conditions for $C^{0}$-viability referring to the systems of the form (1.1).

Remark 3.1. The system (1.1) can be rewritten as a multi-valued nonlinear Cauchy problem in the product space $z=X \times Y$ endowed with the norm $\|\cdot\|_{z}$, defined by $\|(x, y)\|_{Z}=\|x\|_{X}+\|y\|_{Y}$ for each $(x, y) \in z$. Let $\mathcal{A}=(A, B): D(\mathcal{A}) \subseteq \mathcal{Z} \rightarrow \mathcal{Z}$ be defined by $D(\mathcal{A})=D(A) \times D(B), \mathcal{A}(z)=$ $(A x, B y)$ for each $z=(x, y) \in D(\mathcal{A})$ and let $\mathcal{F}: \mathcal{K} \rightsquigarrow \mathcal{Z}$ be the multifunction defined by $\mathcal{F}(t, z)=(F(t, z), G(t, z))=\{(F(t, z), w) ; w \in G(t, z)\}$ for each $(t, z) \in \mathcal{K}$. So, the system (1.1) can be rewritten as

$$
\left\{\begin{array}{l}
z^{\prime}(t) \in \mathcal{A} z(t)+\mathcal{F}(t, z(t)), \quad t \geq \tau \\
z(\tau)=\zeta
\end{array}\right.
$$

where $\zeta=(\xi, \eta)$. Let us remark that, if $\left\{S_{A}: \overline{D(A)} \rightarrow \overline{D(A)} ; t \geq 0\right\}$ and $\left\{S_{B}(t): \overline{D(B)} \rightarrow \overline{D(B)} ; t \geq 0\right\}$ are the semigroups of contractions generated by $A$ and $B$, then $\mathcal{A}$ generates the semigroup $\{\mathcal{S}(t): z \rightarrow z ; t \geq$ $0\}$, given by $\mathcal{S}(t)(x, y)=\left(S_{A}(t) x, S_{B}(t) y\right)$, for each $t \geq 0$ and $(x, y) \in Z$.

We also remark that the graph $\mathcal{K}$ is $C^{0}$-viable with respect to $(A+F, B+$ $G$ ) in sense of Definition 1.2 if and only if $\mathcal{K}$ is $C^{0}$-viable with respect to $\mathcal{A}+\mathcal{F}$ in sense of Definition 2.3, which means that for each $(\tau, \zeta) \in \mathcal{K}$, there exists $T>\tau,[\tau, T] \subseteq I$ such that (3.1) has at least one $C^{0}$-solution $z:[\tau, T] \rightarrow \mathcal{Z}$ with $(t, z(t)) \in \mathcal{K}$ for each $t \in[\tau, T]$.

We introduce the following hypotheses:

$\left(\mathrm{H}_{1}\right) A: D(A) \subseteq X \rightsquigarrow X$ and $B: D(B) \subseteq Y \rightsquigarrow Y$ are $m$-dissipative operators and $\left\{S_{A}(t): \overline{D(A)} \rightarrow \overline{D(A)} ; t \geq 0\right\}$ and $\left\{S_{B}(t): \overline{D(B)} \rightarrow\right.$ $\overline{D(B)} ; t \geq 0\}$ are the nonlinear semigroups of contractions generated by $A$ and $B$ respectively; 
$\left(\mathrm{H}_{2}\right)$ the semigroup $\left\{S_{B}(t): \overline{D(B)} \rightarrow \overline{D(B)} ; t \geq 0\right\}$ is compact, i.e., $S_{B}(t)$ is compact for each $t>0$;

$\left(\mathrm{H}_{3}\right) \quad B: D(B) \subseteq Y \rightsquigarrow Y$ is of compact type;

$\left(\mathrm{H}_{4}\right)$ the graph $\mathcal{K}$ is $\mathcal{A}$ - $C^{0}$-viable by itself;

$\left(\mathrm{H}_{5}\right)$ the graph $\mathcal{K}$ is locally closed from the left;

$\left(\mathrm{H}_{6}\right) F: I \times X \times Y \rightarrow X$ is continuous on $I \times X \times Y$ and locally Lipschitz with respect to its second argument, i.e., for each $(\tau, \xi, \eta) \in$ $I \times X \times Y$ there exist $T>\tau, \rho>0$ and $L=L_{\tau, \xi, \eta}>0$ such that $\|F(t, u, v)-F(t, \widetilde{u}, v)\|_{X} \leq L\|u-\widetilde{u}\|_{X}$ for each $(t, u, v),(t, \widetilde{u}, v) \in$ $[\tau, T] \times D_{X}(\xi, \rho) \times D_{Y}(\eta, \rho) ;$

$\left(\mathrm{H}_{7}\right) G: \mathcal{K} \rightsquigarrow Y$ is essentially locally bounded;

$\left(\mathrm{H}_{8}\right) G: \mathcal{K} \rightsquigarrow Y$ is almost strongly-weakly u.s.c.;

$\left(\mathrm{H}_{9}\right) G: \mathcal{K} \rightsquigarrow Y$ is almost u.s.c.;

$\left(\mathrm{H}_{10}\right)$ there exists a set $N \subseteq I$, with $\lambda(N)=0$ such that for each $(\tau, \xi, \eta) \in$ $[(I \backslash N) \times X \times Y] \cap \mathcal{K}$, we have $(F(\tau, \xi, \eta), G(\tau, \xi, \eta)) \in \mathcal{Q T}_{\mathcal{K}}^{\mathcal{A}}(\tau, \xi, \eta)$.

From Theorem 2.2 and Remark 3.1 we deduce:

Theorem 3.1. Let us assume that $\left(\mathrm{H}_{1}\right)$ is satisfied and $D(A)$ and $D(B)$ are separable. Let $I \subseteq \mathbb{R}$ be a nonempty and open from the right interval, let $K: I \rightsquigarrow \overline{D(A)} \times \overline{\overline{D(B)}}$ be a multi-function with nonempty values and $\mathcal{K}=\operatorname{graph}(\mathrm{K})$. Let $F: \mathcal{K} \rightarrow X$ be a continuous function and let $G: \mathcal{K} \rightsquigarrow Y$ be a multi-function with nonempty and closed values, almost u.s.c. and locally integrally bounded. If $\mathcal{K}$ is $C^{0}$-viable with respect to $(A+F, B+G)$, then $\left(\mathrm{H}_{4}\right)$ and $\left(\mathrm{H}_{10}\right)$ are satisfied.

The next result is the main sufficient condition of $C^{0}$-viability.

Theorem 3.2. Let $X$ and $Y$ be Banach spaces, $I \subseteq \mathbb{R}$ be a nonempty and open from the right interval, let $K: I \rightsquigarrow \overline{D(A)} \times \overline{D(B)}$ be a multifunction with nonempty values and $\mathcal{K}=\operatorname{graph}(\mathrm{K})$. Let us assume that $\left(\mathrm{H}_{1}\right),\left(\mathrm{H}_{2}\right),\left(\mathrm{H}_{3}\right),\left(\mathrm{H}_{4}\right),\left(\mathrm{H}_{5}\right)$ and $\left(\mathrm{H}_{6}\right)$ are satisfied. Let $G: \mathcal{K} \rightsquigarrow Y$ be a nonempty, convex and weakly compact valued multi-function which satisfies $\left(\mathrm{H}_{7}\right)$ and $\left(\mathrm{H}_{8}\right)$. If the tangency condition $\left(\mathrm{H}_{10}\right)$ is satisfied, then $\mathcal{K}$ is $C^{0}$ viable with respect to $(A+F, B+G)$. If $D(A)$ is separable and, instead 
of $\left(\mathrm{H}_{8}\right)$, we assume that $\left(\mathrm{H}_{9}\right)$ is satisfied, then a necessary and sufficient condition in order that $\mathcal{K}$ be $C^{0}$-viable with respect to $(A+F, B+G)$ is $\left(\mathrm{H}_{10}\right)$.

The necessity follows from Theorem 3.1 by observing that $\left(\mathrm{H}_{2}\right)$ implies the separability of $D(B)$, while the sufficiency will be proved in the next section.

\section{Proof of Theorem 3.2}

Proof. From Remark 3.1, it suffices to show that the set $\mathcal{K}$ is $C^{0}$-viable with respect to $\mathcal{A}+\mathcal{F}$. Let $(\tau, \zeta)=(\tau, \xi, \eta) \in \mathcal{K}$ be arbitrary. We will prove that the problem (3.1) has at least one $C^{0}$-solution on an interval $\left[\tau, T_{0}\right]$. Let $Z \subseteq I$ be a negligible set including the negligible sets from $\left(\mathrm{H}_{7}\right)$ and $\left(\mathrm{H}_{10}\right)$. Let $\rho>0, \widetilde{T}>\tau,[\tau, \widetilde{T}] \subseteq I, M_{0}>0, L>0$ and $l_{1} \in L_{\mathrm{loc}}^{\infty}(I ; \mathbb{R})$ be such that $\left([\tau, \widetilde{T}] \times D_{\mathcal{Z}}(\zeta, \rho)\right) \cap \mathcal{K}$ is closed from the left and the next condition are satisfied:

$$
\|F(t, u, v)\|_{X} \leq M_{0}
$$

for each $(t, u, v) \in[\tau, \widetilde{T}] \times D_{X}(\xi, \rho) \times D_{Y}(\eta, \rho)$,

$$
\|F(t, u, v)-F(t, \widetilde{u}, v)\|_{X} \leq L\|u-\widetilde{u}\|_{X}
$$

for each $(t, u, v),(t, \widetilde{u}, v) \in[\tau, \widetilde{T}] \times D_{X}(\xi, \rho) \times D_{Y}(\eta, \rho)$,

$$
\|G(t, u, v)\|_{Y} \leq l_{1}(t)
$$

for each $(t, u, v) \in\left(([\tau, \widetilde{T}] \backslash Z) \times D_{X}(\xi, \rho) \times D_{Y}(\eta, \rho)\right) \cap \mathcal{K}$.

Let $\left(\varepsilon_{n}\right)_{n} \downarrow 0$ be a sequence in $(0,1)$ and let $\left(\mathcal{O}_{n}\right)_{n \geq 1} \subseteq \mathbb{R}$ be a sequence of open sets such that:

(a) $Z \subseteq \mathcal{O}_{n}$ for each $n \geq 1$;

(b) $\mathcal{O}_{n+1} \subseteq \mathcal{O}_{n}$ and $\lambda\left([\tau, T] \cap \mathcal{O}_{n}\right) \leq \varepsilon_{n}$ for each $n \geq 1$;

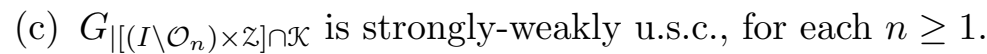

Now, from Necula-Popescu-Vrabie [14, Lemma 5.1], we deduce that there exist $T \in(\tau, \widetilde{T}]$, independent of $n$, and a sequence of $\left(\varepsilon_{n}, \mathcal{O}_{n}\right)$-approximate $C^{0}$-solution of $(3.1)$ on $[\tau, T],\left(\left(\alpha_{n}, \widetilde{f}_{n}, z_{n}\right)\right)_{n}$. This means that, for each $n \geq 1, \alpha_{n}:[\tau, T] \rightarrow[\tau, T]$ is nondecreasing and right continuous, $\widetilde{f}_{n}$ : $[\tau, T] \rightarrow z$ is measurable, $z_{n} \in C([\tau, T] ; z)$ and the following conditions are satisfied. 
(i) $t-\varepsilon_{n} \leq \alpha_{n}(t) \leq t$ for all $t \in[\tau, T], \alpha_{n}(T)=T$;

(ii) for each $t \in[\tau, T]$ for which $\alpha_{n}(t) \in \mathcal{O}_{n}$ it follows that $\left[\alpha_{n}(t), t\right] \subseteq \mathcal{O}_{n}$;

(iii) $z_{n}\left(\alpha_{n}(t)\right) \in D_{\mathcal{Z}}(\zeta, \rho) \cap K\left(\alpha_{n}(t)\right)$ for all $t \in[\tau, T]$;

(iv) $\widetilde{f}_{n}(t) \in \mathcal{F}\left(\alpha_{n}(t), z_{n}\left(\alpha_{n}(t)\right)\right)$ for each $t \in[\tau, T] \backslash \mathcal{O}_{n}$;

(v) $\left\|\widetilde{f}_{n}(t)\right\|_{z} \leq l(t)$ a.e. for $t \in[\tau, T]$, with $l(t)=\max \left\{M_{0}+l_{1}(t), l_{2}(t)\right\}$ where $M_{0}$ is from (4.1), $l_{1} \in L_{\mathrm{loc}}^{\infty}(I ; \mathbb{R})$ is from (4.3) and $l_{2} \in L_{\text {loc }}^{1}(I ; \mathbb{R})$ is as in Definition 2.7;

(vi) $z_{n}(\tau)=\zeta$ and $\left\|z_{n}(t)-u\left(t, \alpha_{n}(s), z_{n}\left(\alpha_{n}(s)\right), \widetilde{f}_{n}\right)\right\|_{z} \leq\left(t-\alpha_{n}(s)\right) \varepsilon_{n}$ for all $t, s \in[\tau, T], \tau \leq s \leq t \leq T$

(vii) $\left\|z_{n}(t)-z_{n}\left(\alpha_{n}(t)\right)\right\|_{z} \leq \varepsilon_{n}$ for all $t \in[\tau, T]$;

$\left(\right.$ viii) $\sup _{t \in[\tau, T]}\|\mathcal{S}(t) \zeta-\zeta\|_{z}+\int_{\tau}^{T} l(s) d s+T-\tau<\rho$.

Let us denote by $\widetilde{f}_{n}:=\left(f_{n}, g_{n}\right)$ and by $z_{n}:=\left(u_{n}, v_{n}\right)$. By (vi), for $s=\tau$, we have

$$
\left\|z_{n}(t)-u\left(t, \tau, \zeta, \widetilde{f}_{n}\right)\right\|_{z} \leq(t-\tau) \varepsilon_{n}
$$

for each $t \in[\tau, T]$, where $u\left(\cdot, \tau, \zeta, \widetilde{f}_{n}\right)$ is the $C^{0}$-solution of the problem

$$
\left\{\begin{array}{l}
z^{\prime}(t) \in \mathcal{A} z(t)+\tilde{f}_{n}(t) \\
z(\tau)=\zeta .
\end{array}\right.
$$

This means that $u\left(\cdot, \tau, \zeta, \widetilde{f}_{n}\right)=\left(x\left(\cdot, \tau, \xi, f_{n}\right), y\left(\cdot, \tau, \eta, g_{n}\right)\right)$ where $x(\cdot)=$ $x\left(\cdot, \tau, \xi, f_{n}\right)$ is the $C^{0}$-solution of the problem

$$
\left\{\begin{array}{l}
x^{\prime}(t) \in A x(t)+f_{n}(t) \\
x(\tau)=\xi
\end{array}\right.
$$

and $y(\cdot)=y\left(\cdot, \tau, \eta, g_{n}\right)$ is the $C^{0}$-solution of the problem

$$
\left\{\begin{array}{l}
y^{\prime}(t) \in B y(t)+g_{n}(t) \\
y(\tau)=\eta
\end{array}\right.
$$


We will prove that, on a subsequence at least, $\left(z_{n}\right)_{n}$ is uniformly convergent on an interval $\left[\tau, T_{0}\right]$ to some function $z$ which is a $C^{0}$-solution of (3.1).

From (i), we obtain

$$
\lim _{n \rightarrow \infty} \alpha_{n}(t)=t
$$

uniformly for $t \in[\tau, T]$.

By (v), we have $\left\|g_{n}(t)\right\|_{Y} \leq l(t)$ for each $n=1,2, \ldots$ and for all $t \in$ $[\tau, T]$ with $l \in L_{\text {loc }}^{1}(I ; \mathbb{R})$. Thus $\left\{g_{n} ; n=1,2, \ldots\right\}$ is uniformly integrable in $L^{1}(\tau, T ; Y)$. Since $B$ generates a compact semigroup, from (4.7), thanks to VRabie [20, Baras' Theorem 2.3.3, p. 47], we conclude that there exists $\widetilde{y} \in C([\tau, T] ; Y)$ such that, on a subsequence at least,

$$
\lim _{n \rightarrow \infty} y\left(t, \tau, \eta, g_{n}\right)=\widetilde{y}(t)
$$

uniformly for $t \in[\tau, T]$. From (4.4) and (4.8), we deduce

$$
\lim _{n \rightarrow \infty} v_{n}(t)=\lim _{n \rightarrow \infty} v_{n}\left(\alpha_{n}(t)\right)=\widetilde{y}(t)
$$

uniformly for $t \in[\tau, T]$. Since, by (iii), we have $v_{n}\left(\alpha_{n}(t)\right) \in D_{Y}(\eta, \rho)$ we deduce $\widetilde{y}(t) \in D_{Y}(\eta, \rho)$ for all $t \in[\tau, T]$.

Now, let us consider the Cauchy problem:

$$
\left\{\begin{array}{l}
x^{\prime}(t)=A x(t)+F(t, x(t), \widetilde{y}(t)), \quad t \in[\tau, T] \\
x(\tau)=\xi
\end{array}\right.
$$

Since $A$ is $m$-dissipative operator, $\xi \in \overline{D(A)}$ and the function $f_{0}:[\tau, T] \times$ $X \rightarrow X, f_{0}(t, x)=F(t, x, \widetilde{y}(t))$ for each $(t, x) \in[\tau, T] \times X$, is continuous on $[\tau, T] \times X$ and locally Lipschitz with respect to $x \in X$, it follows that there exists $T_{0} \in(\tau, T]$ such that the problem (4.11) has an unique $C^{0}$-solution $\widetilde{x}:\left[\tau, T_{0}\right] \rightarrow \overline{D(A)}$.

Let us prove now that $\lim _{n} u_{n}(t)=\widetilde{x}(t)$ uniformly for $t \in\left[\tau, T_{0}\right]$. From (iv) we have $f_{n}(s) \in F\left(\alpha_{n}(s), u_{n}\left(\alpha_{n}(s)\right), v_{n}\left(\alpha_{n}(s)\right)\right)$ for all $s \in\left[\tau, T_{0}\right] \backslash \mathcal{O}_{n}$. From this and (2.2), we deduce

$$
\begin{aligned}
& \left\|x\left(t, \tau, \xi, f_{n}\right)-\widetilde{x}(t)\right\|_{X} \leq \int_{\tau}^{t}\left\|f_{n}(s)-F(s, \widetilde{x}(s), \widetilde{y}(s))\right\|_{X} d s \\
& \leq \int_{[\tau, t] \backslash \mathcal{O}_{n}}\left\|F\left(\alpha_{n}(s), z_{n}\left(\alpha_{n}(s)\right)\right)-F(s, \widetilde{x}(s), \widetilde{y}(s))\right\|_{X} d s
\end{aligned}
$$


$+\int_{[\tau, t] \cap \mathcal{O}_{n}}\left\|f_{n}(s)-F(s, \widetilde{x}(s), \widetilde{y}(s))\right\|_{X} d s$

$\leq \int_{[\tau, t]}\left\|F\left(\alpha_{n}(s), u_{n}\left(\alpha_{n}(s)\right), v_{n}\left(\alpha_{n}(s)\right)\right)-F\left(\alpha_{n}(s), \widetilde{x}(s), v_{n}\left(\alpha_{n}(s)\right)\right)\right\|_{X} d s$

$+\int_{[\tau, t]}\left\|F\left(\alpha_{n}(s), \widetilde{x}(s), v_{n}\left(\alpha_{n}(s)\right)\right)-F(s, \widetilde{x}(s), \widetilde{y}(s))\right\|_{X} d s$

$+\int_{[\tau, t] \cap \mathcal{O}_{n}}\left(\left\|f_{n}(s)\right\|_{X}+\|F(s, \widetilde{x}(s), \widetilde{y}(s))\|_{X}\right) d s$

for each $t \in\left[\tau, T_{0}\right]$. Now, using (4.1), (4.2) and (v), we get

$$
\begin{aligned}
& \left\|x\left(t, \tau, \xi, f_{n}\right)-\widetilde{x}(t)\right\|_{X} \\
& \leq L \int_{\tau}^{t}\left\|u_{n}\left(\alpha_{n}(s)\right)-\widetilde{x}(s)\right\|_{X} d s+2 \int_{[\tau, t] \cap \mathcal{O}_{n}} l(s) d s \\
& +\int_{\tau}^{T_{0}}\left\|F\left(\alpha_{n}(s), \widetilde{x}(s), v_{n}\left(\alpha_{n}(s)\right)\right)-F(s, \widetilde{x}(s), \widetilde{y}(s))\right\|_{X} d s .
\end{aligned}
$$

By (b), (4.8), (4.10) and the continuity of $F$ on $I \times X \times Y$, we deduce that, there exists $\gamma_{n} \downarrow 0$ such that $\int_{\left[\tau, T_{0}\right] \cap \mathcal{O}_{n}} l(s) d s \leq \gamma_{n}$ for $n=1,2, \ldots$ and $\left\|F\left(\alpha_{n}(s), \widetilde{x}(s), v_{n}\left(\alpha_{n}(s)\right)\right)-F((s), \widetilde{x}(s), \widetilde{y}(s))\right\|_{X} \leq \gamma_{n}$ for $n=1,2, \ldots$ and each $s \in\left[\tau, T_{0}\right]$.

On the other hand, we have $\left\|u_{n}\left(\alpha_{n}(t)\right)-\widetilde{x}(t)\right\|_{X} \leq\left\|u_{n}\left(\alpha_{n}(t)\right)-u_{n}(t)\right\|_{X}+$ $\left\|u_{n}(t)-x\left(t, \tau, \xi, f_{n}\right)\right\|_{X}+\left\|x\left(t, \tau, \xi, f_{n}\right)-\widetilde{x}(t)\right\|_{X}$. From (vii), (4.4) and (4.12), we obtain

$$
\left\|u_{n}\left(\alpha_{n}(t)\right)-\widetilde{x}(t)\right\|_{X} \leq \delta_{n}+L \int_{\tau}^{t}\left\|u_{n}\left(\alpha_{n}(s)\right)-\widetilde{x}(s)\right\|_{X} d s,
$$

for each $t \in\left[\tau, T_{0}\right]$, where $\delta_{n}:=\left(1+T_{0}-\tau\right) \varepsilon_{n}+\left(2+T_{0}-\tau\right) \gamma_{n}$. By Gronwall's Lemma, we conclude $\left\|u_{n}\left(\alpha_{n}(t)\right)-\widetilde{x}(t)\right\|_{X} \leq \delta_{n} e^{L\left(T_{0}-\tau\right)}$, for each $n \geq 1$ and each $t \in\left[\tau, T_{0}\right]$. Since $\delta_{n} \rightarrow 0$ and $\alpha_{n}(t) \rightarrow t$ uniformly for $t \in\left[\tau, T_{0}\right]$, we get

$$
\lim _{n \rightarrow \infty} u_{n}\left(\alpha_{n}(t)\right)=\lim _{n \rightarrow \infty} u_{n}(t)=\widetilde{x}(t),
$$

uniformly for $t \in\left[\tau, T_{0}\right]$ and by (4.4),

$$
\lim _{n \rightarrow \infty} x\left(t, \tau, \xi, f_{n}\right)=\widetilde{x}(t),
$$


uniformly for $t \in\left[\tau, T_{0}\right]$. Thus

$$
\lim _{n \rightarrow \infty} z_{n}\left(\alpha_{n}(t)\right)=\lim _{n \rightarrow \infty} z_{n}(t)=\lim _{n \rightarrow \infty} u\left(t, \tau, \zeta, \widetilde{f}_{n}\right)=z(t)
$$

uniformly for $t \in\left[\tau, T_{0}\right]$, where $z:=(\widetilde{x}, \widetilde{y}) \in C\left(\left[\tau, T_{0}\right] ; z\right)$.

Since $z_{n}\left(\alpha_{n}(t)\right) \in K\left(\alpha_{n}(t)\right), \alpha_{n}(t) \uparrow t$ uniformly for $t \in\left[\tau, T_{0}\right]$ and $\left(\left[\tau, T_{0}\right] \times D_{\mathcal{Z}}(\zeta, \rho)\right) \cap \mathcal{K}$ is closed from the left, from (4.15), we deduce that

$$
(t, z(t)) \in \mathcal{K}
$$

for $t \in\left[\tau, T_{0}\right]$.

Let $k \in \mathbb{N}, k \geq 1$ be arbitrary. From (4.8) and (4.15) we deduce that, the set

$$
\overline{\left\{\left(\alpha_{n}(t), z_{n}\left(\alpha_{n}(t)\right)\right) ; t \in\left[\tau, T_{0}\right], n \geq k\right\}}
$$

is compact. Since $\mathcal{F}$ has weakly compact values and $\mathcal{F}_{\mid\left[\left(\left[\tau, T_{0}\right] \backslash \mathcal{O}_{k}\right) \times Z\right] \cap \mathcal{K}}$ is strongly-weakly u.s.c., by CÂRJă-NECULA-VRABIE [6, Lemma 2.6.1, p. 47], it follows that, for each $k \geq 1$, the set

$$
C_{k}:=\overline{\operatorname{conv}}\left(\bigcup_{n \geq k} \bigcup_{t \in\left[\tau, T_{0}\right] \backslash \mathcal{O}_{k}} \mathcal{F}\left(\alpha_{n}(t), z_{n}\left(\alpha_{n}(t)\right)\right)\right)
$$

is weakly compact. From (v) we deduce that $\left\{\widetilde{f}_{n} ; n \geq 1\right\}$ is uniformly integrable in $L^{1}\left(\tau, T_{0} ; Z\right)$ and from (iv) we deduce that, for each $k \geq 1$ and each $n \geq k, \widetilde{f}_{n}(t) \in C_{k}$ for each $t \in\left[\tau, T_{0}\right] \backslash \mathcal{O}_{k}$. Since $C_{k}$ is weakly compact in $z$ and $\lambda\left(\left[\tau, T_{0}\right] \cap \mathcal{O}_{k}\right) \leq \varepsilon_{k}$, by CÂRJĂ-NeCula-Vrabie [6, Diestel's Theorem 1.3 .8$, p. 10], it follows that $\left\{\widetilde{f}_{n}, n \geq 1\right\}$ is weakly relatively compact in $L^{1}\left(\tau, T_{0} ; z\right)$. So, there exists $\tilde{f} \in L^{1}\left(\tau, T_{0} ; z\right)$ such that, on a subsequence at least, $\lim _{n \rightarrow \infty} \widetilde{f}_{n}=\widetilde{f}$ weakly in $L^{1}\left(\tau, T_{0} ; z\right)$. Let us denote by $\widetilde{f}:=(f, g)$.

Since $f_{n}(s)=F\left(\alpha_{n}(s), z_{n}(s)\right)$ for each $s \in\left[\tau, T_{0}\right] \backslash \mathcal{O}_{n}, F$ is a continuous function, $\lim _{n} f_{n}=f$ weakly in $L^{1}\left(\tau, T_{0} ; X\right), \lim _{n}\left(\alpha_{n}(s), z_{n}(s)\right)=(s, z(s))$ uniformly for $s \in\left[\tau, T_{0}\right]$ and $\lim _{n} \lambda\left(\mathcal{O}_{n}\right)=0$, it follows that

$$
f(s)=F(s, \widetilde{x}(s), \widetilde{y}(s))
$$

a.e. for $s \in\left[\tau, T_{0}\right]$. We notice that, in fact, $\lim _{n} f_{n}=f$ strongly in $L^{1}\left(\tau, T_{0} ; X\right)$. Since $g_{n}(s) \in G\left(\alpha_{n}(s), z_{n}\left(\alpha_{n}(s)\right)\right)$ for each $s \in\left[\tau, T_{0}\right] \backslash \mathcal{O}_{k}$, $G_{\mid\left[\left(I \backslash \mathcal{O}_{k}\right) \times Z\right] \cap \mathcal{K}}$ is strongly-weakly u.s.c., $\lim _{n} g_{n}=g$ weakly in $L^{1}\left(\tau, T_{0} ; Y\right)$, $\lim _{n}\left(\alpha_{n}(s), z_{n}(s)\right)=(s, z(s))$ uniformly for $s \in\left[\tau, T_{0}\right]$ and, for each $n \geq k$, 
we have $\alpha_{n}(s) \in\left[\tau, T_{0}\right] \backslash \mathcal{O}_{k}$, from VRABIE [20, Theorem 3.1.2, p. 88], we conclude that $g(s) \in G(s, z(s))$ for each $k \geq 1$ and a.e. for $s \in\left[\tau, T_{0}\right] \backslash \mathcal{O}_{k}$. Since $\lim _{k} \lambda\left(\mathcal{O}_{k}\right)=0$, we conclude that

$$
g(s) \in G(s, \widetilde{x}(s), \widetilde{y}(s))
$$

a.e. for $s \in\left[\tau, T_{0}\right]$. Since $B$ is $m$-dissipative of compact type, $\lim _{n} g_{n}=g$ weakly in $L^{1}\left(\tau, T_{0} ; Y\right)$ and $\lim _{n} y\left(\cdot, \tau, \eta, g_{n}\right)=\widetilde{y}$ strongly in $C\left(\left[\tau, T_{0}\right] ; Y\right)$, we get $\widetilde{y}=y(\cdot, \tau, \eta, g)$. Finally, taking into account that $\widetilde{x}$ is the $C^{0}$-solution of (4.11), from (4.16), (4.17) and (4.18), we conclude that $z=(\widetilde{x}, \widetilde{y})$ is a $C^{0}$-solution of (3.1). The proof is complete.

\section{An example}

Let $H$ be a real Hilbert space, let $C \subseteq H$ be a proper pointed closed convex cone and let " $\preceq$ " be the partial order on $H$ defined by $C$, i.e., $x \preceq y$ if and only if $y-x \in C$. Let $\varphi: H \rightarrow \mathbb{R}_{+} \cup\{\infty\}$ and $\psi: H \rightarrow \mathbb{R}_{+} \cup\{\infty\}$ be two proper, convex and l.s.c. functions and let $\partial \varphi: D(\partial \varphi) \subseteq H \rightsquigarrow H$ and $\partial \psi: D(\partial \psi) \subseteq H \rightsquigarrow H$ be the subdifferentials of $\varphi$ and $\psi$ respectively. Let us denote by $\left\{S_{\varphi}(t): \overline{D(\partial \varphi)} \rightarrow \overline{D(\partial \varphi)} ; t \geq 0\right\}$ and by $\left\{S_{\psi}(t): \overline{D(\partial \psi)} \rightarrow\right.$ $\overline{D(\partial \psi)} ; t \geq 0\}$ the nonlinear semigroups of contractions generated by the $m$-dissipative operators $-\partial \varphi$ and $-\partial \psi$ respectively. Let $a: I \rightarrow D(\partial \varphi)$ and $b: I \rightarrow D(\partial \psi)$ be two continuous functions. Let $K: I \rightsquigarrow H \times H$ be defined $K(t):=\{(x, y) ; a(t) \preceq x, y \preceq b(t)\}$ for each $t \in I$ and let $\mathcal{K}$ be the graph of $K$. Let $F: \mathcal{K} \rightarrow H$ be a given function and $G: \mathcal{K} \rightsquigarrow H$ be a multi-function with nonempty values. Let us consider the problem:

$$
\left\{\begin{array}{l}
u^{\prime}(t) \in-\partial \varphi(u(t))+F(t, u(t), v(t)) \\
v^{\prime}(t) \in-\partial \psi(v(t))+G(t, u(t), v(t)) \\
u(\tau)=\xi, v(\tau)=\eta \\
a(t) \preceq u(t), v(t) \preceq b(t) .
\end{array}\right.
$$

Definition 5.1. A continuous function $(u, v):[\tau, T] \rightarrow D(\partial \varphi) \times D(\partial \psi)$ is a strong solution on $[\tau, T]$ of $(5.1)$ if $(u, v) \in W^{1,2}(\tau, T ; H \times H)$, the function $t \mapsto f(t)=F(t, u(t), v(t))$ belongs to $L^{2}(\tau, T ; H)$ and there exists 
$g \in L^{2}(\tau, T ; H), g(t) \in G(t, u(t), v(t))$ a.e. for $t \in[\tau, T]$ such that:

$$
\left\{\begin{array}{l}
u^{\prime}(t) \in-\partial \varphi(u(t))+f(t), \text { a.e. for } t \in[\tau, T], \\
v^{\prime}(t) \in-\partial \psi(v(t))+g(t), \text { a.e. for } t \in[\tau, T], \\
u(\tau)=\xi, v(\tau)=\eta, \\
a(t) \preceq u(t), v(t) \preceq b(t) \text { for each } t \in[\tau, T] .
\end{array}\right.
$$

Using Theorem 3.2, we obtain a sufficient condition in order that $\mathcal{K}$ be strongly-viable with respect to $(-\partial \varphi+F,-\partial \psi+G)$, i.e., in order that, for each $(\tau, \xi, \eta) \in I \times H \times H$ with $a(\tau) \preceq \xi, \eta \preceq b(\tau)$, the problem (5.1) has at least one strong solution on an interval $[\tau, T]$.

Definition 5.2. A convex function $\psi: H \rightarrow \mathbb{R}_{+} \cup\{\infty\}$ is called of compact type if, for each $k>0$, the level set $\mathcal{L}_{k}=\left\{u \in H ;\|u\|^{2}+\psi(u) \leq k\right\}$ is relatively compact in $H$.

Remark 5.1. If $\psi: H \rightarrow \mathbb{R}_{+} \cup\{\infty\}$ is a proper, convex, l.s.c. function of compact type, then $-\partial \psi$ generates a compact semigroup - see VRABIE [20, Proposition 2.2.2, p. 42],- and it is an $m$-dissipative operator of compact type in the sense of Definition 2.9 - see VRABIE [20, Corollary 2.3.2, p. 50].

Theorem 5.1. Let $H$ be a real Hilbert space, let $\varphi: H \rightarrow \mathbb{R}_{+} \cup\{\infty\}$ and $\psi: H \rightarrow \mathbb{R}_{+} \cup\{\infty\}$ be two proper, convex and l.s.c. functions, with $\partial \varphi$ and $\partial \psi$ single-valued, let $a: I \rightarrow D(\partial \varphi), b: I \rightarrow D(\partial \psi), a, b \in$ $W_{\text {loc }}^{1,1}(I ; H)$, let $C \subseteq \overline{D(\partial \varphi)} \cap \overline{D(\partial \psi)}$ be a closed convex cone with $C \cap$ $(-C)=\{0\}$ and $\overline{D(\partial \varphi) \cap C}=\overline{D(\partial \psi) \cap C}=C$. Let $\mathcal{K}$ be the graph of multi-function $K: I \rightsquigarrow \overline{D(\partial \varphi)} \times \overline{D(\partial \psi)}, K(t)=(a(t)+C, b(t)-C)$ for each $t \in I$. Let us assume that $\psi$ is of compact type, $S_{\varphi}(t) C \subseteq C, S_{\psi}(t) C \subseteq$ $C$ for each $t \in I$ and $\mathcal{K}$ is $(-\partial \varphi,-\partial \psi)-C^{0}$-viable by itself. Let $F$ : $I \times H \times H \rightarrow H$ be a continuous function which is locally Lipschitz with respect to its second argument and $G: \mathcal{K} \rightsquigarrow H$ be a nonempty, convex and weakly compact valued multi-function which is essentially locally bounded and almost strongly-weakly u.s.c. Then, a sufficient condition in order that $\mathcal{K}$ be $C^{0}$-viable with respect to $(-\partial \varphi+F,-\partial \psi+G)$ is to exists a neglijable set $N \subseteq I$ such that, for each $\tau \in I \backslash N$ and each $(\xi, \eta) \in \partial(C \times C) \cap$ $(D(\partial \varphi) \times D(\partial \psi))$, we have

$$
\left\{\begin{array}{l}
\operatorname{dist}\left(\partial \varphi(\xi)-\partial \varphi(a(\tau)+\xi)-a^{\prime}(\tau)+F(\tau, a(\tau)+\xi, b(\tau)-\eta) ; C\right)=0 \\
\operatorname{dist}\left(\partial \psi(\eta)+\partial \psi(b(\tau)-\eta)+b^{\prime}(\tau)-G(\tau, a(\tau)+\xi, b(\tau)-\eta) ; C\right)=0
\end{array}\right.
$$


Proof. We will prove that (5.3) implies the next tangency condition $(F(\tau, a(\tau)+\xi, b(\tau)-\eta), G(\tau, a(\tau)+\xi, b(\tau)-\eta)) \in \mathcal{T S}_{\mathcal{K}}^{(-\partial \varphi,-\partial \psi)}(\tau, a(\tau)+$ $\xi, b(\tau)-\eta)$ or, equivalently

$$
\liminf _{h \downarrow 0} \frac{1}{h} \operatorname{dist}(z(\tau+h, \tau, a(\tau)+\xi, b(\tau)-\eta, E) ; \mathcal{K}(\tau+h))=0
$$

for each $\tau \in I \backslash N$ and each $(\xi, \eta) \in(C \times C) \cap(D(\partial \varphi) \times D(\partial \psi))$, where

$$
\begin{aligned}
& E=(F(\tau, a(\tau)+\xi, b(\tau)-\eta), G(\tau, a(\tau)+\xi, b(\tau)-\eta)) \\
& \quad=\{(x, y) ; x=F(\tau, a(\tau)+\xi, b(\tau)-\eta), y \in G(\tau, a(\tau)+\xi, b(\tau)-\eta)\} \\
& \quad z(\tau+h, \tau, a(\tau)+\xi, b(\tau)-\eta, E) \\
& \quad=\{z(\tau+h, \tau, a(\tau)+\xi, b(\tau)-\eta,(x, y)) ; \quad(x, y) \in E\}
\end{aligned}
$$

and $z(\cdot, \tau, a(\tau)+\xi, b(\tau)-\eta,(x, y))$ is the $C^{0}$-solution of the problem

$$
\left\{\begin{array}{l}
z^{\prime}(t) \in(-\partial \varphi,-\partial \psi) z(t)+(x, y) \\
z(\tau)=(a(\tau)+\xi, b(\tau)-\eta) .
\end{array}\right.
$$

Let us denote by $z(\cdot, \tau, a(\tau)+\xi, b(\tau)-\eta,(x, y)):=(u(\cdot), v(\cdot))$. Then $u(\cdot):=$ $u(\cdot, \tau, a(\tau)+\xi, x)$, where $x=F(\tau, a(\tau)+\xi, b(\tau)-\eta)$, is a $C^{0}$-solution of

$$
\left\{\begin{array}{l}
u^{\prime}(t) \in-\partial \varphi(u(t))+x \\
u(\tau)=a(\tau)+\xi
\end{array}\right.
$$

and $v(\cdot):=v(\cdot, \tau, b(\tau)-\eta, y)$, where $y \in G(\tau, a(\tau)+\xi, b(\tau)-\eta)$, is a $C^{0}$ solution of

$$
\left\{\begin{array}{l}
v^{\prime}(t) \in-\partial \psi(v(t))+y \\
v(\tau)=b(\tau)-\eta
\end{array}\right.
$$

Let us denote by $O_{j}, j=1,2, \ldots$ some functions defined on $(0,1)$ with values in $H$, with $\lim _{h \downarrow 0} O_{j}(h)=0$.

Let $h \in(0,1), \xi \in C \cap D(\partial \varphi), \eta \in C \cap D(\partial \psi)$ such that $a(\tau)+\xi \in D(\partial \varphi)$ and $b(\tau)-\eta \in D(\partial \psi)$. Let us denote by $x=F(\tau, a(\tau)+\xi, b(\tau)-\eta)$ and let $y \in G(\tau, a(\tau)+\xi, b(\tau)-\eta)$ be arbitrary but fixed. We have

$$
\left\{\begin{array}{l}
a(\tau+h)=a(\tau)+h a^{\prime}(\tau)+h O_{1}(h) \\
b(\tau+h)=b(\tau)+h b^{\prime}(\tau)+h O_{1}(h),
\end{array}\right.
$$




$$
\left\{\begin{array}{l}
u(\tau+h, \tau, a(\tau)+\xi, x)=a(\tau)+\xi-h \partial \varphi(a(\tau)+\xi)+h x+h O_{2}(h) \\
v(\tau+h, \tau, b(\tau)-\eta, y)=b(\tau)-\eta-h \partial \psi(b(\tau)-\eta)+h y+h O_{2}(h)
\end{array}\right.
$$

and

$$
\left\{\begin{array}{l}
S_{\varphi}(h) \xi=\xi-h \partial \varphi(\xi)+h O_{3}(h) \\
S_{\psi}(h) \eta=\eta-h \partial \psi(\eta)+h O_{3}(h),
\end{array}\right.
$$

where $\left\{S_{\varphi}(t): \overline{D(\partial \varphi)} \rightarrow \overline{D(\partial \varphi)}, t \geq 0\right\}$ and $\left\{S_{\psi}(t): \overline{D(\partial \psi)} \rightarrow \overline{D(\partial \psi)}, t \geq\right.$ $0\}$ are the nonlinear semigroups generated by $-\partial \varphi$ and $-\partial \psi$ respectively.

Since $\xi \in C \cap D(\partial \varphi), \eta \in C \cap D(\partial \psi), S_{\varphi}(h) C \subseteq C, S_{\psi}(h) C \subseteq C$ and $C$ is a convex cone, it follows that

$$
C \subseteq-S_{\varphi}(h) \xi+C, \quad C \subseteq-S_{\psi}(h) \eta+C \text { and } h C=C .
$$

In view of (5.8), (5.9), (5.10) and (5.11) we get

$$
\begin{aligned}
& \operatorname{dist}(u(\tau+h, \tau, a(\tau)+\xi, x) ; a(\tau+h)+C) \\
& =\operatorname{dist}\left(a(\tau)+\xi-h \partial \varphi(a(\tau)+\xi)+h x+h O_{2}(h) ; a(\tau)+h a^{\prime}(\tau)+h O_{1}(h)+C\right) \\
& =\operatorname{dist}\left(\xi-S_{\varphi}(h) \xi-h \partial \varphi(a(\tau)+\xi)-h a^{\prime}(\tau)+h x+h O_{4}(h) ;-S_{\varphi}(h) \xi+C\right) \\
& =\operatorname{dist}\left(h \partial \varphi(\xi)-h \partial \varphi(a(\tau)+\xi)-h a^{\prime}(\tau)+h x+h O_{5}(h) ;-S_{\varphi}(h) \xi+C\right) \\
& \leq \operatorname{dist}\left(h \partial \varphi(\xi)-h \partial \varphi(a(\tau)+\xi)-h a^{\prime}(\tau)+h x+h O_{5}(h) ; C\right) \\
& \leq \operatorname{dist}\left(h \partial \varphi(\xi)-h \partial \varphi(a(\tau)+\xi)-h a^{\prime}(\tau)+h x+h O_{5}(h) ; h C\right) .
\end{aligned}
$$

So, we have

$$
\begin{aligned}
& \operatorname{dist}(u(\tau+h, \tau, a(\tau)+\xi, x) ; a(\tau+h)+C) \\
& \leq h \operatorname{dist}\left(\partial \varphi(\xi)-\partial \varphi(a(\tau)+\xi)-a^{\prime}(\tau)+x ; C\right)+h\left\|O_{5}(h)\right\| .
\end{aligned}
$$

Similarly we deduce

$$
\begin{aligned}
& \operatorname{dist}(v(\tau+h, \tau, b(\tau)-\eta, y) ; b(\tau+h)-C) \\
& =\operatorname{dist}\left(b(\tau)-\eta-h \partial \psi(b(\tau)-\eta)+h y+h O_{2}(h) ; b(\tau)+h b^{\prime}(\tau)+h O_{1}(h)-C\right) \\
& =\operatorname{dist}\left(\eta-S_{\psi}(h) \eta+h \partial \psi(b(\tau)-\eta)+h b^{\prime}(\tau)-h y+h O_{6}(h) ;-S_{\psi}(h) \eta+C\right) \\
& =\operatorname{dist}\left(h \partial \psi(\eta)+h \partial \psi(b(\tau)-\eta)+h b^{\prime}(\tau)-h y+h O_{7}(h) ;-S_{\psi}(h) \eta+C\right) \\
& \leq \operatorname{dist}\left(h \partial \psi(\eta)+h \partial \psi(b(\tau)-\eta)+h b^{\prime}(\tau)-h y+h O_{7}(h) ; C\right) \\
& \leq \operatorname{dist}\left(h \partial \psi(\eta)+h \partial \psi(b(\tau)-\eta)+h b^{\prime}(\tau)-h y+h O_{7}(h) ; h C\right) .
\end{aligned}
$$


So, we have

$$
\begin{aligned}
& \operatorname{dist}(v(\tau+h, \tau, b(\tau)-\eta, y) ; b(\tau+h)-C) \\
& \leq h \operatorname{dist}\left(\partial \psi(\eta)+\partial \psi(b(\tau)-\eta)+b^{\prime}(\tau)-y ; C\right)+h\left\|O_{7}(h)\right\| .
\end{aligned}
$$

On the other hand, we have

$$
\begin{aligned}
& \operatorname{dist}(z(\tau+h, \tau, a(\tau)+\xi, b(\tau)-\eta, E) ; \mathcal{K}(\tau+h)) \\
& \leq \operatorname{dist}(u(\tau+h, \tau, a(\tau)+\xi, x) ; a(\tau+h)+C) \\
& +\operatorname{dist}(v(\tau+h, \tau, b(\tau)-\eta, y) ; b(\tau+h)-C) .
\end{aligned}
$$

Dividing by $h$ and passing to the limit for $h \downarrow 0$ in (5.14) and using (5.12) and (5.13), we get

$$
\begin{aligned}
& \liminf _{h \downarrow 0} \frac{1}{h} \operatorname{dist}(z(\tau+h, \tau, a(\tau)+\xi, b(\tau)-\eta, E) ; \mathcal{K}(\tau+h)) \\
& \leq \operatorname{dist}\left(\partial \varphi(\xi)-\partial \varphi(a(\tau)+\xi)-a^{\prime}(\tau)+x ; C\right) \\
& +\operatorname{dist}\left(\partial \psi(\eta)+\partial \psi(b(\tau)-\eta)+b^{\prime}(\tau)-y ; C\right)
\end{aligned}
$$

for each $y \in G(\tau, a(\tau)+\xi, b(\tau)-\eta)$, where $x=F(\tau, a(\tau)+\xi, b(\tau)-\eta)$.

If $(\xi, \eta) \in \partial(C \times C) \cap(D(\partial \varphi) \times D(\partial \psi))$, we have

$$
\begin{aligned}
& \operatorname{dist}\left(\partial \psi(\eta)+\partial \psi(b(\tau)-\eta)+b^{\prime}(\tau)-G(\tau, a(\tau)+\xi, b(\tau)-\eta) ; C\right) \\
& =\inf \left\{\operatorname{dist}\left(\partial \psi(\eta)+\partial \psi(b(\tau)-\eta)+b^{\prime}(\tau)-y ; C\right) ;\right. \\
& \quad y \in G(\tau, a(\tau)+\xi, b(\tau)-\eta)\}
\end{aligned}
$$

and, by (5.3) and (5.15) we conclude that (5.4) holds true.

If $(\xi, \eta) \in((C \times C) \backslash \partial(C \times C)) \cap(D(\partial \varphi) \times D(\partial \psi))$ then, for $h>0$ small enough, we have $\operatorname{dist}(z(\tau+h, \tau, a(\tau)+\xi, b(\tau)-\eta, E) ; \mathcal{K}(\tau+h))=0$. So (5.4) holds true for each $(\xi, \eta) \in(C \times C) \cap(D(\partial \varphi) \times D(\partial \psi))$ and this completes the proof.

Remark 5.2. Since $F$ is single-valued, $G$ is a convex and weakly compact valued multi-function and $C$ is convex and closed, (5.3) is equivalent to: for each $\tau \in I \backslash N$ and each $(\xi, \eta) \in \partial(C \times C) \cap(D(\partial \varphi) \times D(\partial \psi))$, there exists $y \in G(\tau, a(\tau)+\xi, b(\tau)-\eta)$ such that

$$
\left\{\begin{array}{l}
\partial \varphi(\xi)-\partial \varphi(a(\tau)+\xi)-a^{\prime}(\tau)+x \in C \\
\partial \psi(\eta)+\partial \psi(b(\tau)-\eta)+b^{\prime}(\tau)-y \in C,
\end{array}\right.
$$

where $x=F(\tau, a(\tau)+\xi, b(\tau)-\eta)$. 
Acknowledgements. This work was supported by PN-II-ID-PCE2007-1, Grant ID_397.

\section{REFERENCES}

1. Barbu, V. - Nonlinear Semigroups and Differential Equations in Banach Spaces, Editura Academiei, Bucureşti, Noordhoff, 1976.

2. Bothe, D. - Flow invariance for perturbed nonlinear evolution equations, Abstr. Appl. Anal., 1 (1996), 417-433.

3. Bothe, D. - Multivalued perturbations of $m$-accretive differential inclusions, Israel J. Math., 108 (1998), 109-138.

4. BURLICĂ, M. - Viability for semi-multi-valued reaction-diffusion systems, CP1048 Numerical Analysis and Applied Mathematics, International Conference 2008, edited by T. E. Simos, G. Psihoyios and Ch. Tsitouras, AIP, 126-129.

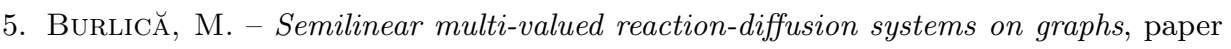
in preparation.

6. CÂrJă, O.; Necula, M.; Vrabie, I.I. - Viability, Invariance and Applications, North-Holland Mathematics Studies, 207, Elsevier, 2007.

7. CÂrJă, O.; Necula, M.; Vrabie, I.I. - Necessary and sufficient conditions for viability for nonlinear evolution inclusions, Set-Valued Anal, 16 (2008), 701-731.

8. CÂrJă, O.; Necula, M.; Vrabie, I.I. - Necessary and sufficient conditions for viability for semilinear differential inclusions, Trans. Amer. Math. Soc., 361 (2009), 343-390.

9. CÂRJă, O.; Necula, M.; VRABIE, I.I. - Tangent sets, viability for differential inclusions and applications, Nonlin. Anal., DOI:10.1016/j.na.2009.01.055.

10. CÂRJĂ, O.; VRABIE, I.I. - Viability results for nonlinear perturbed differential inslusions, Panamer. Math. J., 9 (1999), 63-74.

11. CÂRJĂ, O.; VRABIE, I.I. - Viability for semilinear differential inclusions via the weak sequential tangency condition, J. Math. Anal. Appl., 262 (2001), 24-38.

12. Lakshmikantham, V.; Leela, S. - Nonlinear Differential Equations in Abstract Spaces, International Series in Nonlinear Mathematics, 2, Pergamon Press, 1981.

13. Necula, M.; Popescu, M.; VRabie, I.I. - Evolution equations on locally closed graphs and applications, Nonlin. Anal., Proceedings of WCNA-2008, submitted.

14. Necula, M.; Popescu, M.; VRabie, I.I. - Nonlinear evolution equations on locally closed graphs, Rev. R. Acad. Cien. Serie A. Mat., in print.

15. PAVEL, N.H. - Invariant sets for a class of semi-linear equations of evolution, Nonlinear Anal., 1 (1977), 187-196. 
16. PAVEL, N.H.; VRABIE, I.I. - Équations d'évolution multivoques dans des espaces de Banach, C.R. Acad. Sci. Paris Sér A-B, 287 (1978), 315-317.

17. Pavel, N.H.; VRABIE, I.I. - Semilinear evolution equations with multivalued righthand side in Banach spaces, An. Ştiinţ. Univ. "Al.I. Cuza" Iaşi Secţ. I a Mat., 25 (1979), 137-157.

18. RoşU, D. - Viability for nonlinear multi-valued reaction-diffusion systems, CP1048 Numerical Analysis and Applied Mathematics, International Conference 2008, edited by T. E. Simos, G. Psihoyios and Ch. Tsitouras, AIP, 458-461.

19. VRABIE, I.I. - Compactness methods and flow-invariance for perturbed nonlinear semigroups, An. Ştiinţ. Univ. "Al.I. Cuza" Iaşi, Secţ. I a, Mat., 27 (1981), 117-125.

20. VRABIE, I.I. - Compactness methods for nonlinear evolutions, Second Edition, Pitman Monographs and Survey in Pure and Applied Mathematics, 75, Longman Scientific Technical, New York, 1995.

Received: 7. VIII.2009

Department of Mathematics,

"Gh.Asachi" University,

Bvd. Carol I., 700506, Iaşi,

ROMANIA

rosudaniela100@yahoo.com 